\title{
Mobiles and Cognition: The Associations Between Mobile Technology and Cognitive Flexibility
}

\author{
https://doi.org/10.3991/ijim.v14i03.11233 \\ Arhondoula Alexopoulou ${ }^{(凶)}$, Alexandra Batsou, Athanasios Drigas \\ N.C.S.R. "Demokritos", Athens, Greece \\ dollyalexopoulou@gmail.com
}

\begin{abstract}
Mobile phones hold a rather permeating role in our lives. Their portability and ease of use have turned them to indispensable parts of our everyday activities, since they are steadily connected to the internet, providing a wide range of applications at the same time. However, there has been little research so far, on the way mobile technology habits could be related to alterations in our cognitive functioning. The review presented in this paper, introduces findings of the last decade on the field of cognitive flexibility, some of which, tend to be contradictory at times. Cognitive flexibility is presented through the prism of attention-switching, task-switching and in close relation to working memory and inhibitory control, functions which have been proved to be interrelated. Moving a step forward, an attempt was made to present a more contemporary approach towards cognitive flexibility and the ways it is affected by mobile and advanced information and communication technologies.
\end{abstract}

Keywords - Cognitive flexibility, mobile phones, ICT, task-switching, working memory, inhibition control, attention control, multitasking, social networking sites (SNS), self-training, search engines.

\section{Introduction}

Mobile technology and use are nowadays ubiquitous in modern individuals' habits and identity, as they have become part of their culture, modifying how all sorts of activities and social interactions are carried out. Eonta et al. [1], aptly characterise mobile technology as omnipresent, comprising a kind of pervasive integration into users' lives. As Garcia-Mondes, Cabballero-Munoz and Perez-Alvarez [2] point out, modern human needs a wide variety of information to function properly. Since mobile phones are fully embedded in the everyday routine of the individuals, certain alterations in the way this routine is carried out can be mentioned. For instance, SMS messaging has largely substituted phone calls. Social interactions of today's adolescents and adults take place online, mostly on mobiles, since their transferability and ease of use are undeniable. Individuals use mobile phone- provided calculators, navigators, reminders, note pads, alarm clocks, and search engines, to make their daily routine easier. Moving a step forward, Miller [3] supports that mobile use enhances positively and even reconstructs a person's psychology, to a greater extent than the use of PCs. As Howells et al. [4] 
indicate in the same direction, it has been proved through research that a great range of simple cognitive and behavioural strategies, known as positive interventions, can make a person happier. Therefore, the development of mobile phone-based content can positively influence people and is considered to appeal to the range of strategies mentioned above. Indeed, using only basic mobile features, which can be downloaded easily, a mobile phone can be transformed into a powerful means of intervention. This power of intervention is evolving dynamically, yet it still needs to be investigated, as mobile phones can be considered as behaviour or even identity shaping devices. There even seems to occur a re-definition of time perception, perceived as a limitation of the span of action to a brief course, absolutely determined by the use of a mobile device.

Connections between mobile phone technology, ICTs and cognition have been established, to a certain degree. Numerous surveys and publications on mobile phones and ICTs usage and their effects on learning exist, however, equivalent ones concerning direct effects on cognitive functions, are of small scale and are mostly based on learning outcome surveys. Far from trying to review or question every psychological impact of mobile technology advancements, or any endeavour to investigate all possible technological breakthroughs that are relevant to psychology, we are inclined to a more contemporary approach towards cognitive flexibility; one that fits into mobile and advanced information and communication technologies.

\section{How is Cognitive Flexibility Defined}

In psychology, flexible is considered the person who can use his skills to deal with a new problem or task, which is not similar to the ones that he has been trained for. Consequently, a flexible person must be ready to 'switch' from one course of action to another without any delay, showing the cognitive ability to adapt his behaviour according to the requirements of the new context [5]. An individual's cognitive flexibility plays a truly crucial role in their organizational ability to comply with changing environmental demands [6]. It is also essential for finding solutions to different problems and being creative, so, it is normally defined through terms of attention-switching, taskswitching, set-shifting and inhibition control. It could also be divided into response flexibility and representational flexibility, sometimes also referred to as attentional flexibility, according to Zelazo and Frye [7]. The most appropriate definition, however, is probably the one by Canas, Quesada, Antoli, and Fajardo [8], who define cognitive flexibility as the human ability to reformulate the cognitive processing strategies according to new and unexpected conditions in the environment. Cognitive flexibility has been recognized as a key antecedent of behaviour in particularly complex and unstructured tasks [9], which is related to the higher cortical functions and controls the way people feel, think and act. It is included among the executive brain functions (EF), together with motor and interference inhibition, performance monitoring, attention control, planning, decision-making, temporal foresight and working memory [10]. In order to behave flexibly, an individual ought to think and act in a similarly flexible way. However, it is not an ability possessed from the early stages of life, since it is closely related to higher levels of integration, of different brain functions. It develops together 
with maturity, declines in senility [11], and is enabled by the enforcement of working memory and inhibitory control. Therefore, task switching requires the involvement of more executive functions [12], for the process to be completed. As it has been shown through research, cognitive flexibility is not considered as a constant or non-developing function. Indeed, there are variations in an individual's cognitive flexibility, even from moment to moment [13]. Concerning task switching, it has to be pointed out that it is a key element of cognitive flexibility, the study of which comprises quite an active field of research, both in cognitive neuroscience and in experimental psychology [6].

Cognitive flexibility needs control; therefore, it needs the ability to hold back immediate reactions and promote delayed responses [14]. It has been proved by research that, from an early age until adulthood, in addition to cognitive maturation, task-related areas of the brain are gradually triggered and lead to the higher area of control, of the executive functions. Once these brain regions are fully developed, mature and controlled cognitive functions are formulated [15]. Moving a step further, Canas, Fajardo, and Salmeron [16] consider cognitive flexibility as a learning process acquired through experience. According to them, it involves the ability of an individual to adapt to new or unexpected situations. This adaptation is not always successful since the automation of skill is essential to claim that cognitive flexibility has been achieved.

\section{Differentiated Instruction to Several Skills Through the Use of Mobile Phones}

Scientists are now at the point of concurrent progress of research and mobile phone applications, since mobiles keep on creating and supporting a great amount of behavioural data. Participants in neuropsychological types of assessment are often tested in everyday environments with the use of mobile devices, as researchers want to achieve performance in real situations, avoiding laboratories, where testing conditions are characterised by limited distractions and helpful laboratory staff. Timmers et al. [17] confirmed in their research, that young healthy participants were able to keep focused attention on task-relevant information, simultaneously avoiding any distraction coming from the surroundings. The data of their research also proved that the participants' short-term memory was enhanced, up to a degree. The research of Brown et al. [18] was oriented in the same direction. According to the results of the research, the qualities were mentioned with accuracy and they were comparable to laboratory measurements, thus proving a mass collection of research data via smartphones quite efficient.

Bless et al. [19] used mobile phones in a slightly different direction, developing a mobile application based on self-administered cognitive training. Self-training has been considered of great importance, since individuals can be trained at a convenient place and time, away from the stressful environment of a laboratory. They wanted to investigate the efficacy of using a mobile application for self-supervised auditory attention training, focusing on whether training on domains of attention and transfer of the trained task to other tasks would be successful. Regarding the fMRI findings, task trans- 
fer to similar tasks was not observed, although the opposite was expected. The researchers attributed it to the small sample of the research and to the fact that the training task proved to be rather too specific to be transferred.

\section{$4 \quad$ Media Multi-Tasking and Task Switching}

Frequent mobile phone users are engaged in activities relevant to task switching, every time they are involved with social sites, send text messages or listen to music, on the same or different devices, at the same time. This is what is called "multitasking", i.e. doing many different tasks, simultaneously. Current studies show that the people who can 'multitask' create a different brain from the rest of the people. Ophir, Nass, and Wagner [20], after conducting a relevant to the subject research, supported that heavy media multitaskers had difficulty in task switching. According to them, extensive practice with switching back and forth between different tasks reduces the ability to switch properly. Urged by these findings, Alzahabi and Becker [21] conducted their research, only to come up with entirely different results. They used two different methods, one of which was an exact reproduction of Ophir et al. [20]. They support the fact that heavy media multitaskers have developed an ability to process different sources of information simultaneously, thus limiting switching needs. Due to extensive practice on task switching, heavy users seemed to have the ability to adapt to new tasks by inhibiting previous ones; consequently, task-switching costs were confined.( In order to define a 'switch cost', Arrington and Logan [22] describe an experimental procedure, during which the subjects of research are called to accomplish various tasks, in response to given stimuli. The reaction time (RT) needed to respond between tasks of a similar type, is faster than the reaction time required to respond between tasks of an altered or an entirely different type. This reaction time difference is called a task switching cost).

As far as the dual-task performing was concerned, extensive practice produced nonimpressive results; after all dual-task performance ensues from single-task performance. Accomplishing a task for the first time demands more time and attention than repeating the same task. Even through task repetition, no interference from other nonsignificant tasks was observed. Thus, media multitasking proved to be relevant to high task reconfiguration ability. Concerning this reconfiguration ability, Alzahabi and Becker [21] claimed that successful transferring between trained and new tasks is determined by how similar the tasks are. Consequently, this constraint seemed to be reduced for frequent media users, as they proved to be better at task reconfigurations, resulting in generalisations more easily. Moving a step further, based on a few other pieces of research, they supported that media multitasking does not associate with limitation in attentional control. Rosen, Lim, Carrier, and Cheever [23] also reached the same conclusion in their research. They pointed out, that young individuals who are accustomed to multitasking and continuous communication via their mobile phones, consciously make use of metacognitive strategies to multitask and do equally well at all tasks. Alzahabi and Becker [21] attributed the dissonance between their research and the one of Ophir et al. [20], to the fact that the years between the two pieces of research were significant enough for a rapid change in the media landscape; ICT and mobile 
phones users increased in number and their engagement with multiple forms of media altered the way they multitasked. Consequently, the way multitasking induces changes in cognitive functions, has changed. According to them, however, more research is needed to shed light on the impact and the long-term consequences of multitasking on cognition.

\section{$5 \quad$ Adoption of New Technologies Towards Flexible Thinking and Learning}

Studies have shown that regular and repetitive mental stimulation results in improvements in cognition, even in older people, who have decreased cognitive abilities [11]. According to their research, personalized computerized cognitive training provides individuals with significant cognitive benefits. Given that learning supported and provided by various technological means has been an efficient common practice nowadays, it is reasonable to make use of those means to train or enhance a person's cognitive flexibility. Concerning the learning processes themselves, since they evolve and are constantly reconfigured, they require individuals to be more flexible and open- minded at adopting both new technologies and multiple ways of accessing information. Autonomous and active learning is thus facilitated, according to Barak and Levenberg [24], who concluded that flexible thinking comprises of learning new technologies acceptance, open-mindedness, and adaptation to new contexts. They also confirmed that these qualities are interrelated and act reciprocally. Spiro, Collins, and Ramchandran had already stated that openness to new technologies and flexibility are interdependent [25]. However, there are significant differences in the way people perceive and use mobiles and new technologies. They claim that flexibility based on being open and receptive results in being able to adapt and correctly apply knowledge to new situations.

So far, researches on the field of cognition indicate that prolonged exposure to technology and media devices probably has a great impact on cognition, significantly affecting the way our brain remembers or processes information. Abramson et al. [26] examined cognitive functions in a study called MoRPhEUS, aiming to investigate the cognitive effects of mobile phone exposure, in young adolescents and school children. They concluded that more frequent users showed smaller task-switching costs on associative learning tasks, however, their working memory seemed to work less accurately. There was also some evidence of deterioration on the inhibitory function. According to them, the faster reaction times can be attributed to the innate tendency of young individuals to respond fast; accuracy comes second. The researchers, however, do not exclude the possibility of applying learned behaviours due to the frequent use and they agree on the need for future research on the field.

On the other hand, Sayan [27] proved in his research, that mobiles and mobile apps such as WhatsApp, can be used as goal-achieving tools, affecting and enhancing students' learning ability. Spiro et al. [25] were largely occupied with an individual's learning ability and the factors that determine it. They placed great importance on someone being receptive, that is, open and eager to learn. They concluded through their re- 
search, that being a receptive learner, applies to various ways of learning through hypertext environments, based on cognitive flexibility theory. They stress the importance of being receptive as substantial to the final shaping of an individual's knowledge, through the consolidation of appropriate mind habits, characterised by accuracy in responding to situations, interrelatedness and diversified reproduction. A few years earlier and in a rather preliminary attempt, Granic and Lamey [28] had also considered hypertext technology, as cognitive flexibility enhancing. Hypertext environments, such as the World Wide Web, provide knowledge in the form of multiple heterogeneous contexts. A single search may provide the user with a great number of different sites and relevant or not hyperlinks, varying one to another in opinion, standards, and validity. The user then needs to be effective in terms of being flexible; flexibility connected to hypertext environments can be defined as flexibility in formulating proper searches and competence at switching among tasks i.e. among searches until the search is finetuned. As a result, the cognitive gap is filled. Jonassen et al. [29] share the same opinion and consider hypertexts as a natural medium to acquire information. According to them, hypertext environments support and enhance searches within authentic, informationrich contexts. Cognitive flexibility hypertexts provide multiple representations of different contents, based on the fact that the real world is complex; consequently, learners should be trained to perceive knowledge and function through the instruction of more complex and multiple patterns.

\section{Social Networking Sites and Search Engines - Mobile Activity on the Internet and New Flexibility Traits}

Undeniably, the internet is a multiple-level environment, on which many different life actions are realised. Individuals proceed on all sorts of activities having to do with teaching and learning, shopping, taking up hobbies, working and socialising. The use of social networking sites (SNS) has dramatically increased in the last years, demanding more and more of an individual's cognitive resources [30]. Social networking sites users are often required to make simultaneous use of several digital technologies, especially mobile phones, on which they are called to multitask, sharing their attention among different tasks. Working memory, bears the burden of updating information, mental shifting among several tasks and inhibiting the information which is no longer needed, to accomplish these tasks. As far as attentional control is concerned, the synergy of inhibition control, shifting and information updating in working memory is required, to be achieved. According to the results of Alloway and Alloway research [30], probably the use of SNS alters the way attention is assigned. Active users, after fulfilling the sustained attention test, they were given, were proved to allocate equal attention to every incoming stimulus. Passive users, on the other hand, seemed to target their attention to a single piece of information and inhibit others. However, with practice, they were able to automatise their attentional skills. Both categories of users were quite accurate in their responses. Facebook users seem to simultaneously turn all comments and updates, to useful networking data, instead of inhibiting them. Checking friends' status updates on Facebook requires working memory training since the user needs first 
to update and then keep the current information. Concerning activity on YouTube and video recommendations to others, it is equally connected to working memory, according to them. Users are interested in maintaining their best public image, so they carefully select videos to recommend them to their peers, obviously taking into consideration their preferences and likes. This also involves adaptation of memory to several different situations, given that working memory is usually enabled with the help of constant task performance and the use of the information the brain receives [31]. As it can easily be understood, users of SNS seem flexible enough to handle, process and make beneficial, for them, the use of social networks. Alloway and Alloway [30], believe however, that further research is needed to confirm their results, especially concerning long-term benefits for cognition, resulting from SNS activities.

Social networking sites activities are accomplished on the internet, which is an indispensable part of the function of a mobile phone. Mobile internet user penetration worldwide, has risen from $48.8 \%$, in 2014, to $63.4 \%$, in the first months of 2019 [32]. Bearing in mind the aforementioned figures and the fact that the internet is not only used for entertainment but also as a means of providing information, it can easily be deduced that mobile phone searches on Google and other search engines have become synonyms of quick and easy access to any kind of information, filling all kinds of knowledge gaps. Search engines, especially Google, which is the one mostly used, have become a kind of human external memory. Sparrow, Liu, and Wegner [33] investigated the statement above and also examined whether human's internal ability to encode information is developed towards the source and the way of retrieving it, rather than maintaining the information itself. Users proved to be more interested in the availability of the information, as they placed primacy on the source of retrieving information, relying on the data stored on search engines. Barr, Pennycook, Stolz, and Fugelsang [34] also claim, as a result of their research, that there is possibly a relation between heavy users of mobile phones and negative effects on analytic thinking since individuals use their phones as a source of memory, which is always at hand. Granic and Lamey [28] hold a different perspective on the matter, considering the shifts on an individual's cognition from a different point of view. The Internet follows a more complex, abstract, non-linear pattern of organizing information. Users are called to cope with this kind of interactive information presented in the form of hypertext, which is different from the linear text at hand presentation of the previous century. It seems that it is not a matter of analytic but rather a matter of flexible thinking.

\section{Conclusion}

Cognitive flexibility needs to be reconsidered as a dynamic process, acquired with maturation and training and characterised by self-regulation and adaptability to new situations. Throughout this paper, there has been an attempt to demonstrate the positive and negative findings related to the way and the extent mobile technologies affect cognitive flexibility. Undeniably, mobile phones serve as powerful computers always at hand. This characteristic, first of all, turns them to tools of differentiated instruction to 
several skills. They have been used for self-instructed and supervised cognitive training, through special software. Mobile phones have also served as a useful, innovative tool for collecting great amounts of behavioural data, in neuropsychological types of assessment. Consequently, mobile technology enables users to more differentiated instruction to several skills, demanding higher cognitive abilities at the same time [27].

Frequent mobile users are characterised by multitasking, on and via their devices. Multitaskers are considered to develop different cognitive abilities. Although the first researches on the field showed that multitaskers had some difficulty in task switching [20], later duplicate researches came with different results. Multitasking seems to enhance the ability of switching from one task to another, by minimizing task-switching costs, resulting in generalisations and therefore maintaining better cognitive control skills [21]. An individual needs to consciously make use of metacognitive strategies to monitor and regulate himself every time multitasking is involved, to gain the best performance and adaptation to all sorts of tasks, i.e. to achieve cognitive flexibility [23]. However, further research including longitudinal evaluation is necessary, to fully comprehend the relationship between media multitasking, high or mediocre mobile phone use and cognitive flexibility.

People undergoing the right training have the chance to improve themselves at certain tasks. When this training is implemented through new technologies, individuals are provided with significant cognitive benefits, even at older ages [11]. Flexibility on being open and receptive results in correct use of knowledge together with adaptability to new situations [25]. Being receptive, also applies to ways of learning through hypertext environments, after all, hypertext technology is considered as flexibility enhancing [29], [28] and [25]. Cognitive flexibility theory serves as a model to design learning environments, enabling both the acquisition of advanced knowledge and its use in complex and multiple settings. Flexibility connected to hypertext environments can be defined as competence at formulating and reformulating searches and at task switching, i.e. among searches. Any kind of openness achieved through cognitive flexibility hypertexts, aims at mind-shifting from the closed stereotypes to more open ones, simultaneously shaping knowledge by making correlations, to apply it to many different situations. This leads to the formation and the reoccurrence of more complex, diversified and flexible ways of thinking. After all, the internet has been ranked as a self-organising system, which can impose major shifts in the user's cognition [28]. There exists an interactive kind of relationship, in which, not only does the user employ technology for his own purposes, but technology also alters the user's way of thinking, according to its operational set of rules.

SNS users seem to have developed the ability to update, retain and reuse all sorts of incoming information. This also involves working memory adaptation to different situations [31], [30] and the ability to make generalisations. All these abilities are indicative of cognitive flexibility. However, some researchers consider that working memory is degraded because of the extensive use of search engines on the internet [34], [33]. Considering the belief above from a different perspective, working memory seems to be directed in a twofold way; not only to maintain but also to retrieve information. Individuals need to employ the ability to formulate and reformulate a search, a skill which is demanding in terms of memory, attention and inhibition control. This can be 
translated, not as a debilitation of working memory or even of long-term memory but as a different, flexible 'meta-function' of memory, bearing new qualities and potential, fully adjacent to the new technological era. After all, the users of the last decade are the ones who have a 'digital mother tongue', therefore they are well acquainted with all these functions that researchers are striving to explain and categorize.

Cognitive flexibility has been reconsidered in the present review, under the prism of sustained attention, information processing, task- switching, and working memory. All these executive functions can be perceived as one unified meta-ability [35], to handle and maintain the information, plan and execute tasks, focus attention and inhibit behaviour, in mobile phone environments. Indisputably, further research and studies are needed to investigate in-depth all possible effects of mobile phone use on cognitive flexibility and the other executive functions as well, since they act separately but not independently.

\section{References}

[1] Eonta, A. M., Christon, L. M., Hourigan, S. E., Ravindran, N., Vrana, S. R., \& SouthamGerow, M. A. (2011). Using everyday technology to enhance evidence-based treatments. Professional Psychology: Research and Practice, 42(6), 513. https://doi.org/10.1037/a0025 $\underline{825}$

[2] García-Montes, J. M., Caballero-Munoz, D., \& Perez-Alvarez, M. (2006). Changes in the self- resulting from the use of mobile phones. Media, Culture \& Society, 28(1), 67-82. https://doi.org/10.1177/0163443706059287

[3] Miller, G. (2012). The smartphone psychology manifesto. Perspectives on psychological science, 7(3), 221-237.

[4] Howells, A., Ivtzan, I., \& Eiroa-Orosa, F. J. (2016). Putting the 'app' in happiness: a randomised controlled trial of a smartphone-based mindfulness intervention to enhance wellbeing. Journal of Happiness Studies, 17(1), 163-185. https://doi.org/10.1007/s10902-014$\underline{9589-1}$

[5] Stemme, A., Deco, G., \&Busch, A. (2007). The neuronal dynamics underlying cognitive flexibility in set shifting tasks. Journal of computational neuroscience, 23(3), 313. https:// doi.org/10.1007/s 10827-007-0034-X

[6] Kiesel, A., Steinhauser, M., Wendt, M., Falkenstein, M., Jost, K., Philipp, A. M., \& Koch, I. (2010). Control and interference in task switching-A review. Psychological bulletin, 136(5), 849. https://doi.org/10.1037/a0019842

[7] Zelazo, P. D., \& Frye, D. (1998). Cognitive complexity and control: II. The development of executive function in childhood. Current Directions in Psychological Science, 7(4), 121126. https://doi.org/10.1111/1467-8721.ep10774761

[8] Canas, J., Quesada,J., Antoli, A.,\& Fajardo, I.(2003) Cognitive flexibility and adaptability to environmental changes in dynamic complex problem-solving tasks. Ergonomics, 46(5), 482-501. https://doi.org/10.1080/0014013031000061640

[9] Cohen, J.D., McClure,S. M., \&Yu,A.J. (2007) Should I stay or should I go? How the human brain manages the trade-off between exploitation and exploration. Philosophical Transactions of the Royal Society B: Biological Sciences, 362 (1481), 933-942. https:// doi.org/10.1098/rstb.2007.2098 
[10] Stuss, D. T., \& Alexander, M. P. (2000). Executive functions and the frontal lobes: a conceptual view. Psychological research, 63(3-4), 289-298. https://doi.org/10.1007/s0042699 $\underline{00007}$

[11] Peretz, C., Korczyn, A. D., Shatil, E., Aharonson, V., Birnboim, S., \& Giladi, N. (2011). Computer-based, personalized cognitive training versus classical computer games: a randomized double-blind prospective trial of cognitive stimulation. Neuroepidemiology, 36(2), 91-99. https://doi.org/10.1159/000323950

[12] Logan, G. D. (2004). Working memory, task switching, and executive control in the task span procedure. Journal of Experimental Psychology: General, 133(2), 218. https://doi. org/10.1037/0096-3445.133.2.218

[13] Leber, A.B., Turk-Browne, N.B., Chun M.M. (2008). Neural predictors of moment-to-moment fluctuations in cognitive flexibility. Proceedings of the National Academy of Sciences Sep 2008, 105 (36) 13592-13597 https://doi.org/10.1073/pnas.0805423105

[14] Laureiro-Martínez, D., Brusoni, S., \& Zollo, M. (2009). Cognitive flexibility in decisionmaking: A neurological model of learning and change. CROMA-Center for Research in Organization and Management-Bocconi University, 1, 1-43.

[15] Rubia, K. (2013). Functional brain imaging across development. European child \& adolescent psychiatry, 22(12), 719-731. https://doi.org/10.1007/s00787-012-0291-8

[16] Canas, J. J., Fajardo, I., \& Salmeron, L. (2006). Cognitive flexibility. International encyclopedia of ergonomics and human factors, 1, 297-301.

[17] Timmers, C., Maeghs, A., Vestjens, M., Bonnemayer, C., Hamers, H., \& Blokland, A. (2014). Ambulant cognitive assessment using a smartphone. Applied Neuropsychology: Adult, 21(2), 136-142. https://doi.org/10.1080/09084282.2013.778261

[18] Brown, H. R., Zeidman, P., Smittenaar, P., Adams, R. A., McNab, F., Rutledge, R. B., \& Dolan, R. J. (2014). Crowdsourcing for cognitive science-the utility of smartphones. PloS one, 9(7), e100662. https://doi.org/10.1371/journal.pone.0100662

[19] Bless, J. J., Westerhausen, R., Kompus, K., Gudmundsen, M., \& Hugdahl, K. (2014). Selfsupervised, mobile-application based cognitive training of auditory attention: a behavioral and fMRI evaluation. Internet Interventions, 1(3), 102-110. https://doi.org/10.1016/j.invent.2014.06.001

[20] Ophir, E., Nass, C., \& Wagner, A. D. (2009). Cognitive control in media multitaskers. Proceedings of the National Academy of Sciences of the Unites States of America, 106, 15,58315,587. https://doi.org/10.1073/pnas.0903620106

[21] Alzahabi, R., \& Becker, M. W. (2013). The association between media multitasking, taskswitching, and dual-task performance. Journal of Experimental Psychology: Human Perception and Performance, 39(5), 1485. https://doi.org/10.1037/a0031208

[22] Arrington, C. M., \& Logan, G. D. (2004). The cost of a voluntary task switch. Psychological science, 15(9), 610-615. https://doi.org/10.1111/j.0956-7976.2004.00728.x

[23] Rosen, L. D., Lim, A. F., Carrier, L. M., \& Cheever, N. A. (2011). An empirical examination of the educational impact of text message-induced task switching in the classroom: Educational implications and strategies to enhance learning. Psicología educativa, 17(2), 163-177. https://doi.org/10.5093/ed2011v17n2a4

[24] Barak, M., \& Levenberg, A. (2016). Flexible thinking in learning: An individual differences measure for learning in technology-enhanced environments. Computers \& Education, 99, 39-52. https://doi.org/10.1016/j.compedu.2016.04.003

[25] Spiro, R. J., Collins, B. P., \& Ramchandran, A. R. (2008). Modes of openness and flexibility in cognitive flexibility hypertext learning environments. In Online and Distance Learning: Concepts, Methodologies, Tools, and Applications (pp. 1903-1908). IGI Global. https://doi.org/10.4018/978-1-59904-935-9.ch152 
[26] Abramson, M. J., Benke, G. P., Dimitriadis, C., Inyang, I. O., Sim, M. R., Wolfe, R. S., \& Croft, R. J. (2009). Mobile telephone use is associated with changes in cognitive function in young adolescents. Bioelectromagnetics, 30(8), 678-686. https://doi.org/10.1002/bem. 20534

[27] Sayan, H. (2016). Affecting higher students learning activity by using WhatsApp. European Journal of Research and Reflection in Educational Sciences Vol, 4(3).

[28] Granic, I., \& Lamey, A. V. (2000). The self-organization of the Internet and changing modes of thought. New Ideas in psychology, 18(1), 93-107. https://doi.org/10.1016/s0732$\underline{118 x(99) 00039-2}$

[29] Jonassen, D. H., Dyer, D., Peters, K., Robinson, T., Harvey, D., King, M., \& Loughner, P. (1997). Cognitive flexibility hypertexts on the Web: Engaging learners in meaning making. Web-based instruction, 119-133.

[30] Alloway, T. P., \& Alloway, R. G. (2012). The impact of engagement with social networking sites (SNSs) on cognitive skills. Computers in Human Behavior, 28(5), 1748-1754. https://doi.org/10.1016/j.chb.2012.04.015

[31] Guse, B., Falkai, P., \& Wobrock, T. (2010). Cognitive effects of high-frequency repetitive transcranial magnetic stimulation: a systematic review. Journal of neural transmission, 117(1), 105-122. https://doi.org/10.1007/s00702-009-0333-7

[32] (C) Statista, Mobile phone internet user penetration worldwide from 2014 to 2019, (n.d.), retrieved from: https://www.statista.com/statistics/284202/mobile-phone-internet-user-penetration-worldwide/

[33] Sparrow, B., Liu, J., \& Wegner, D. M. (2011). Google effects on memory: Cognitive consequences of having information at our fingertips. Science, 333(6043), 776-778. https:// doi.org/10.1126/science. 1207745

[34] Barr, N., Pennycook, G., Stolz, J. A., \& Fugelsang, J. A. (2015). The brain in your pocket: Evidence that Smartphones are used to supplant thinking. Computers in Human Behavior, 48, 473-480. https://doi.org/10.1016/j.chb.2015.02.029

[35] Terras, M. M., \& Ramsay, J. (2012). The five central psychological challenges facing effective mobile learning. British Journal of Educational Technology, 43(5), 820-832. https://doi.org/10.1111/j.1467-8535.2012.01362.x

\section{$9 \quad$ Authors}

A. Alexopoulou is with the Institute of Informatics and Telecommunications - Net Media Lab \& Mind-Brain R\&D, Agia Paraskevi, 153 10, Athens, Greece (e-mail: dollyalexopoulou@gmail.com).

A. Batsou is with the Institute of Informatics and Telecommunications - Net Media Lab \& Mind-Brain R\&D, Agia Paraskevi, 153 10, Athens, Greece (e-mail: alexmbat@yahoo.gr).

A. Drigas is a Research Director at N.C.S.R. 'Demokritos', Institute of Informatics and Telecommunications - Net Media Lab \& Mind-Brain R\&D, Agia Paraskevi, 153 10, Athens, Greece (e-mail: dr@iit.demokritos.gr).

Article submitted 2019-07-09. Resubmitted 2019-12-13. Final acceptance 2019-12-19. Final version published as submitted by the authors. 